\title{
SUB MANDIBULECTOMY FOR CHRONIC SCLEROSING SIALADENITIS - COMMENTS ON A CLINICAL CASE
}

\author{
Mihail TUSALIU1,2凶, Iulia TITA ${ }^{1}$, Lavinia G. ILINCA ${ }^{1}$, Cristina M. GOANTA ${ }^{2,3}$, \\ Florentin A. VREJU ${ }^{4}$, Octavian D. PALADE ${ }^{5}$
}

1 „Prof. Dr. D. Hociota“ Institute of Phonoaudiology and Functional ENT Surgery, Bucharest, Romania

2 "Carol Davila“ University of Medicine and Pharmacy, Bucharest, Romania

3 „Sf. Pantelimon“ Clinical Emergency Hospital, Bucharest, Romania

${ }^{4}$ Craiova University of Medicine and Pharmacy, Department of Rheumatology, Craiova, Romania

${ }^{5}$ Grigore T. Popa" University of Medicine and Pharmacy, Second Surgery Department, Iasi, Romania

Received 23 Sept 2019, Accepted 02 Nov 2019

https://doi.org/10.31688/ABMU.2019.54.4.23

\section{Abstract}

Introduction. Sialolithiasis is one of the most common pathologies of the salivary glands. When calculi are present in the distal excretory duct, in the hilum or in the parenchyma, complete removal of the submandibular gland is recommended.

Case report. We present the case of a 43-year-old male patient, suffering from left chronic submandibular sialadenitis. The patient accused recurring colic and intermittent swelling of the left submandibular gland experienced for about 10 years, with a progressive evolution. CT scan revealed a lower volume of the left submandibular gland compared to the right submandibular gland, the presence of a microcalcification in the parenchyma and an oval calcification corresponding to the projection area of the left submandibular gland's duct. A left sub mandibulectomy was performed.

Conclusion. Complete removal of the submandibular gland is the most effective therapy for sialolithiasis, being associated with better long-term outcomes and low operative morbidity.

\section{Résumé}

Sous-mandibulectomie pour sialadénite chronique sclérosante - commentaires sur un cas clinique

Introduction. La sialolithiase est une des plus communes pathologies des glandes salivaires. Lorsque les calculs sont présents dans le canal excréteur distal, dans le hile ou dans le parenchyme, l'ablation complète des glandes sous-mandibulaires est recommandée.

Rapport de cas. On présente le cas d'un patient de sexe masculin, âgé de 43 ans, souffrant de sialadénite sous-mandibulaire chronique gauche. Le patient accusait des coliques récurrentes et une tuméfaction intermittente de la glande sous-mandibulaire gauche, expérimentés depuis environ 10 ans, avec une évolution progressive. La tomodensitométrie a révélé un volume réduit de la glande sous-mandibulaire gauche par rapport à la glande sous-mandibulaire droite, La présence d'une microcalcification dans le parenchyme et d'une calcification ovale correspondant à la région de projection du canal de la glande sous-mandibulaire gauche. Une sous-mandibulectomie gauche a été réalisée. 
Keywords: submandibular lithiasis, surgical treatment, salivary glands.

\section{INTRODUCTION}

The submandibular glands are a pair of salivary glands located within the digastric triangle, inferior and posterior to the mandibular body. Each of the submandibular glands consists of a large, superficial lobe and a smaller, deep lobe. It is the second largest, major salivary gland ${ }^{1}$. In a typical adult, the submandibular excretory duct or Wharton's duct measures approximately $5 \mathrm{~cm}$ in length and 2 to $3 \mathrm{~mm}$ in diameter, with a considerably thinner wall compared to the other salivary secretory ducts.

Sialolithiasis is one of the most common pathologies of the salivary glands ${ }^{1,2}$, as well as the most common cause of unilateral diffuse swelling. It represents the most frequent reason for performing a submandibular gland excision and $80 \%$ of all sialolithiasis cases occur within the submandibular gland ${ }^{3}$. While the exact pathogenesis of calculi formation remains unknown ${ }^{2}$, the accumulation of specific conditions, such as long narrow duct, slow saliva flow going upstream against gravity, a high content of calcium, and metabolic processes that increase the amount of alkaline and viscous secretions in the oral cavity, are all believed to favor submandibular gland calculi formation ${ }^{4}$.

Sialolithiasis typically causes symptoms associated with salivary colic, such as intermittent pain accompanied by an acute swelling in the region of the salivary gland or duct. It generally occurs during mealtimes, due to the obstruction in saliva flow, and gradually disappears within the next 2-3 hours. Salivary stasis favors the development of retrograde secondary bacterial infections, which in turn can lead to the formation of salivary abscesses. Recurrent and chronic infection and inflammation can cause chronic sclerosing sialadenitis, giving the submandibular gland a pseudo-tumoral aspect ${ }^{1,5}$.

Common imaging examination methods used for diagnosis in cases with suspicion of inflammatory pathologies of the parenchyma or the ducts of the submandibular salivary glands include: radiography, sialography, ultrasonography, sialo-endoscopy, computed tomography and magnetic resonance imaging.
Conclusion. L'ablation complète de la glande sous-mandibulaire est le traitement le plus efficace contre la sialolithiase, car il est associé à de meilleurs résultats à long terme et à une morbidité opératoire réduite.

Mots-clés: lithiase sous-mandibulaire, traitement chirurgical, glandes salivaires.

Unenhanced computed tomography is often used as a primary screening method ${ }^{6,7}$.

For differential diagnosis, pathologies with similar clinical features include: acute and chronic sialadenitis, submandibular adenopathies, branchial and dermoid cysts, benign and malignant tumours, cellulitis or abscess of the submandibular area, submandibular adenophlegmon, tuberculosis, syphilis and actinomycosis of the submandibular gland ${ }^{1,4,8}$.

Treatment options include various surgical techniques and antibiotic treatment in case of infection, combined with local hygiene?

\section{Case report}

In this paper, the authors present the case of a 43-year-old male patient, suffering from left chronic sclerosing sialadenitis. The patient accused recurring colic and intermittent swelling of the left submandibular gland, symptoms he reported to have experienced for about 10 years, with a progressive evolution. Clinical examination of the submandibular gland revealed no signs outside of typical parameters, as did the laboratory results. A computer tomography examination was performed. The left submandibular gland had a lower volume compared to the right submandibular gland, revealing the presence of a 0.5 $\mathrm{cm}$ microcalcification in the parenchyma and an oval calcification of $1 / 0.6 \mathrm{~cm}$ corresponding to the projection area of the left submandibular gland's duct. The aspect suggests excretory duct lithiasis of the left submandibular gland with upstream glandular atrophy (Figure 1).

Performing a Sebileau - Carrega left cervical incision under general anesthesia, the subcutaneous planes were detached, exposing and then dissecting the platysma muscle and the superficial cervical fascia. The left submandibular gland was exposed, exhibiting a sclero-atrophic appearance and a firm, inhomogeneous consistency (Figure 2).

Dissection, ligature and sectioning of the left facial vein were then performed (Figure 3).

The upper lateral part of the gland coming in contact with the upper side of the digastric muscle was dissected. The gland was detached from the adjacent 


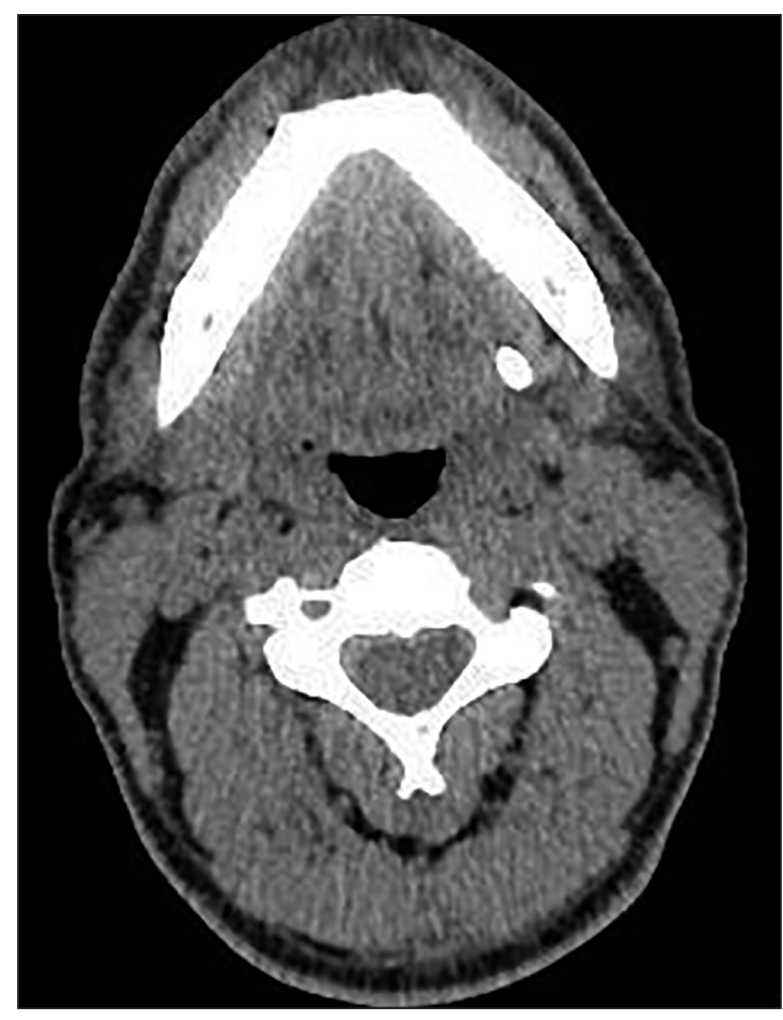

Fig. 1. CT aspect with left submandibular intraductal lithiasis

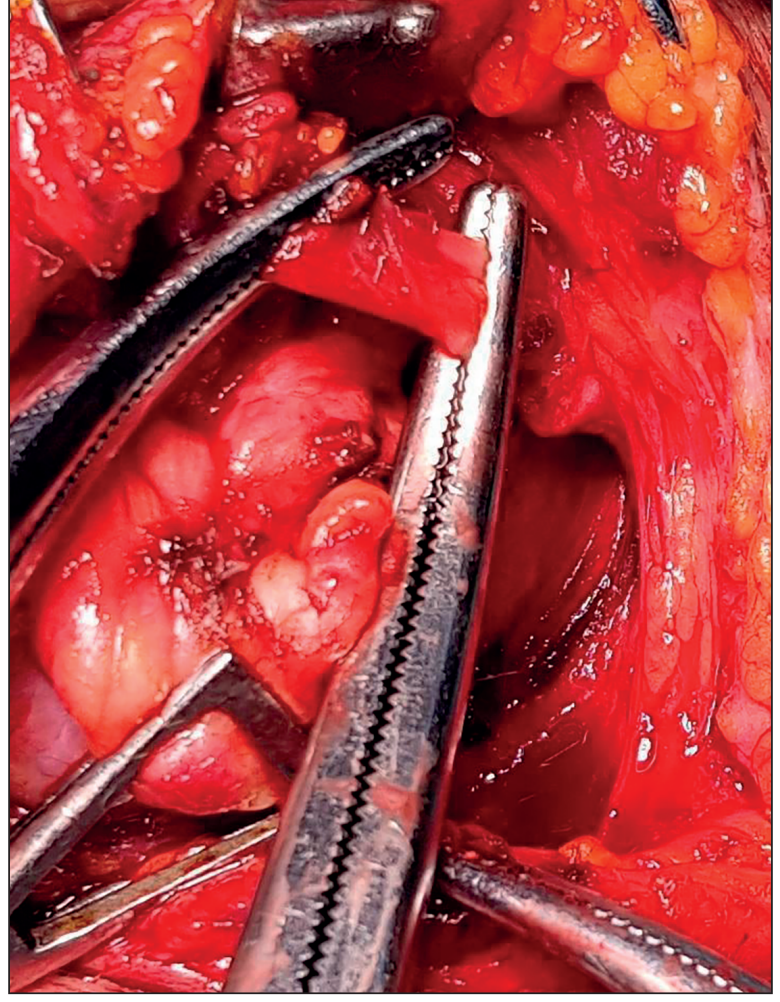

Fig. 2. Intraoperative aspect of the left submandibular gland

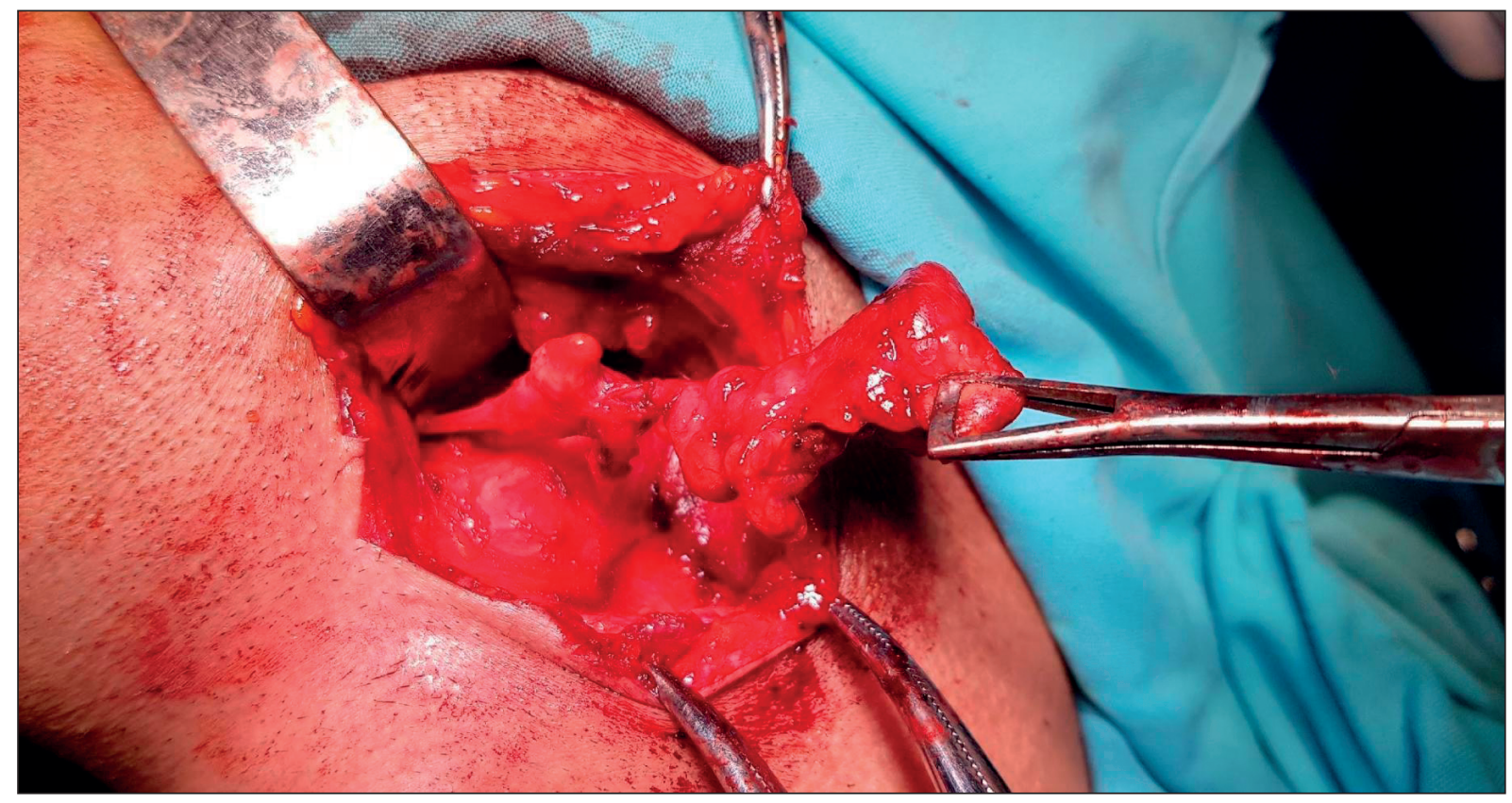

Fig. 3. Left facial vein ligature

submandibular tissue. Dissecting towards the posterior part of the gland, the left lingual nerve, intimately adherent to the Wharton's duct, was exposed. Dissection and release of the nerve from the peri nervous adhesions were then carried out. Wharton's duct was dissected, exposing an intraductal calculus.
The duct was examined by bimanual palpation, followed by ligature and sectioning of Wharton's duct (Figure 4).

A left submandibulectomy was performed, the excised material being referred to histopathologic examination (Figure 5). 


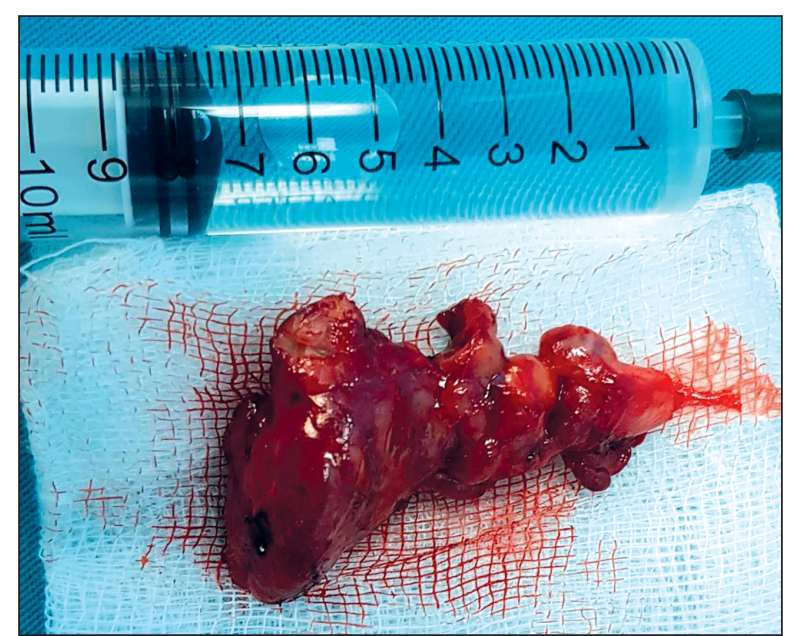

Fig. 4. Exposure, ligature and section of left Wharton's duct

Post-surgery, the patient's healing process exhibited an overall positive general evolution, after intravenous antibiotics, nonsteroidal anti-inflammatory and antiallergic agents.

\section{Discussion}

Sialolithiasis constitutes the most common reason for performing a submandibular gland excision. The diagnosis of sialolithiasis is carried out based on clinical presentation, reported symptoms and imaging techniques. In the process of establishing a positive and differential diagnosis, various techniques can be employed. Radiographic examinations can detect the presence of radio-opaque stones, accounting for about $80 \%$ of all calculi. As diagnostic tests, CT scan represents the most convenient one especially for large calculi. Having a higher sensitivity, unenhanced computed tomography is often used as a primary screening tool, being considered the best widely available method for detecting sialolithiasis. However, it has the disadvantage of not allowing a precise calculus localization. Sialography, involving the use of a contrast agent to highlight the morphology of the duct, is a technique used in detecting radiolucent calculi, or when trying to avoid the superimposition of bone over the areas of interest. Sialography is still considered to be the "gold standard" investigation method for submandibular lithiasis because it allows a precise localization of the calculi and of the glandular ducts architecture. Also, diagnostic sialo-endoscopy is a method that is increasingly used as it allows for the most detailed exploration of the glandular ductal system. Ultrasonography and magnetic resonance imaging generally yield inferior results to computer tomography in terms of detecting

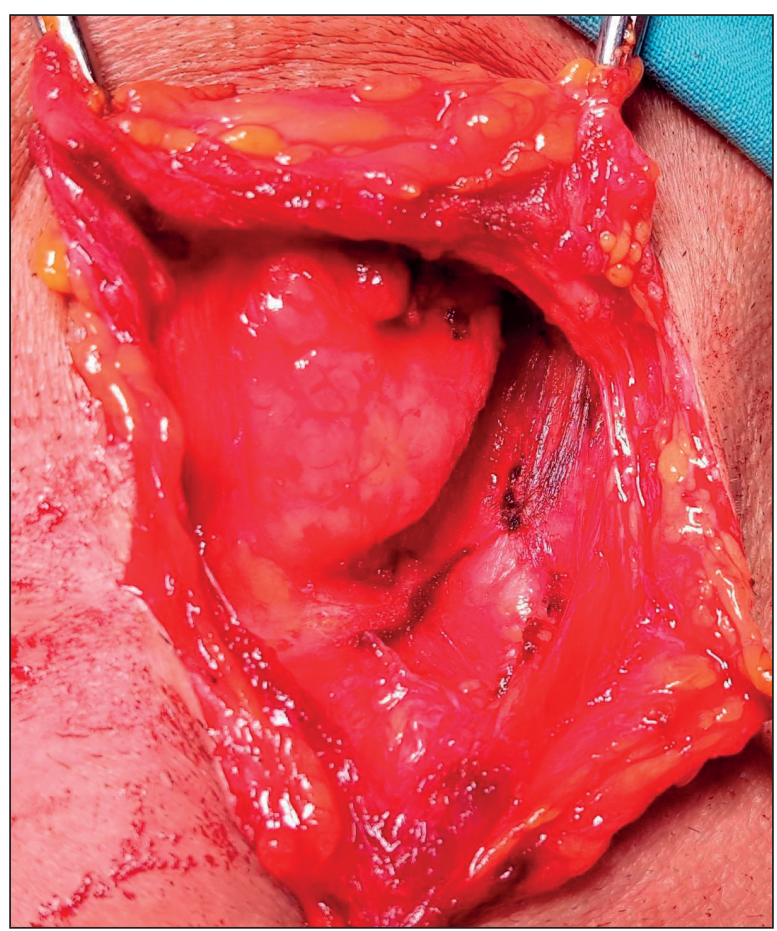

Fig. 5. Excised left submandibular gland

calcifications. Scintigraphy can be used to measure the secretory function. The use of endoscopic and minimally invasive techniques, such as sialo-endoscopy, both a diagnosis and therapeutic method, allows for greater preservation of the gland.

Recommended initial treatment includes improved local hygiene, adequate hydration, oral administration of anti-staphylococcal antibiotics and repeated massaging of the submandibular gland after tenderness becomes less severe, in order to stimulate the flow of saliva. Surgery continues to be a mainstay in the treatment of sialolithiasis, but conservative approaches are increasingly being used in the treatment of this pathology. Extracorporeal shockwave lithotripsy is used for treating fixed calculi. Basket extraction is performed when small, mobile calculi are present. Intraoral surgery is the preferred course of action when calculi are located in the peripheral part of the submandibular duct. Transoral surgery is used when the calculi are present in the proximal Wharton's duct, thus, in this case, it is possible to spare the gland. Complete removal of the gland is recommended when calculi are present in the distal excretory duct, in the hilum or in the parenchyma ${ }^{3,10}$.

Possible complications that could arise from performing a submandibulectomy may include salivary fistulas or sialoceles, nerve paralysis or paresis, hematoma, wound infections, inflammations caused by residual lithiasis in the salivary duct, aesthetic sequelae and hypertrophic scars ${ }^{11,12}$. 
In terms of patient's medical history, he reported starting to experience symptoms characteristic of sialolithiasis approximately 10 years prior to presenting himself to our clinic. Repeated episodes of acuteness, together with periodic calculi formation determine a chronic sclerotic transformation of the submandibular gland, which presents a pseudo tumoral aspect. The computed tomography examination suggested the diagnosis of excretory duct lithiasis with upstream glandular atrophy and highlighted the exact location of the calculus within the left submandibular gland. The histopathologic examination confirmed the result. When the calculus is present in the distal part of Wharton's duct, sub maxillectomy is the recommended treatment. Consequently, the authors opted to pursue this course of action for the presented case. In order for the patient to retain normal post-surgery face muscle functions, the marginal mandibular branch of the facial nerve needs to be preserved. Post-surgery, the patient managed to preserve symmetrical function and full control of the facial nerve, showing no signs of impairment. Aesthetically, the result was satisfactory, with excess scar tissue successfully avoided. A follow-up examination performed one month after surgery revealed no new detectable mass upon palpation.

The therapeutic management of submandibular lithiasis is still not clearly established. The distinctiveness of the presented case is the long clinical evolution (over 10 years) with recurrent episodes of left submandibular lithiasis, repeated inflammatory spurts and attenuated infections. These led to a chronic sclerotic transformation of the gland with a pseudo-tumoral aspect and non-functionality. For this case, the optimal therapeutic solution was left submandibulectomy and histopathological exam of the excision piece.

\section{Conclusion}

Complete removal of the submandibular gland is the most effective therapy for sialolithiasis, being associated with better long-term outcomes and low operative morbidity.

\section{Compliance with Ethics Requirements:}

„The authors declare no conflict of interest regarding this article"

"The authors declare that all the procedures and experiments of this study respect the ethical standards in the Helsinki Declaration of 1975, as revised in 2008(5), as well as the national law. Informed consent was obtained from the patient included in the study"

"No funding for this study"

\section{References}

1. Holsinger FC, Bui DT. Anatomy, function, and evaluation of the salivary glands. Salivary Gland Disorders. Springer, Berlin, Heidelberg. 2007:1-16.

2. Mimura M, Tanaka N, Ichinose S, Kimijima Y, Amagasa T. Possible etiology of calculi formation in salivary glands: biophysical analysis of calculus. Medical Molecular Morphology. 2005;38(3):189-195.

3. Escudier MP, Brown JE, Drage NA, McGurk M. Extracorporeal shockwave lithotripsy in the management of salivary calculi. British Journal of Surgery. 2003; 90(4):482-485.

4. Waseem BZ, Forte V. An unusual case of bilateral submandibular sialolithiasis in a young female patient. International Journal of Pediatric Otorhinolaryngology, 2005;69(5):691-694.

5. Probst R, Grevers G, Iro H. The salivary glands: a step-by-step learning guide. Thieme, Stuttgart, 2005:131-151.

6. Rzymska-Grala I, Stopa Z, Grala B, et al. Salivary gland calculi-contemporary methods of imaging. Polish Journal of Radiology. 2010;75(3):25.

7. Loevner LA, Battineni ML. Imaging of the salivary glands. Salivary Gland Diseases Surgical and Medical Management. Thieme, Stuttgart, 2006:16-26.

8. Bull TR, Thieme S. Color Atlas of ENT Diagnosis, 4th edition, Thieme, 2003:238.

9. Kopeć T, Wierzbicka M, Szyfter W, Leszczyńska M. Algorithm changes in treatment of submandibular gland sialolithiasis. European Archives of Oto-Rhino-Laryngology. 2013;270(7):2089-2093.

10. Theissig J, Rettinger G, Werner JA. ENT-Head and Neck Surgery: Essential Procedures, Thieme, Stuttgart, 2011:302-305.

11. Diaconu C, Balaceanu A, Morosan E. Sepsis biomarkers: past, present and future. Farmacia 2015;63(6):811-815.

12. Tica OA, Tica O, Antal L, et al. Modern oral anticoagulant treatment in patients with atrial fibrillation and heart failure: insights from the clinical practice. Farmacia 2018;66(6):972-976. 